\title{
Characteristics of magnetocardiography and electrocardiography in the time-frequency domain
}

\author{
LIU XinYuan ${ }^{1 *}$, PEI LiuQing ${ }^{2}$, ZHANG SuMing ${ }^{1}$, WANG Yin ${ }^{1} \&$ DAI YuanDong ${ }^{3}$ \\ ${ }^{1}$ School of Electronics Engineering and Computer Science, Peking University, Beijing 100871, China; \\ ${ }^{2}$ College of Information Science and Technology, Beijing Normal University, Beijing 100875, China; \\ ${ }^{3}$ State Key Laboratory for Artificial Microstructure and Mesoscopic Physics, School of Physics, Peking University, Beijing 100871, China
}

Received November 23, 2009; accepted April 23, 2010

\begin{abstract}
As the carriers of electromagnetic information about the cardiac system, both magnetocardiography (MCG) and electrocardiography (ECG) are non-stationary signals (NSS). The essential and crucial features of NSS are the local time-frequency characteristics (LTFCH). Under the constraint of the uncertainty principle in the time-frequency domain (TFD), the LTFCH of those non-stationary signals can be determined by evolutionary spectrum analysis (ESA). In this paper, by analyzing the QRS complexes of both MCG and ECG data from healthy volunteers, we have derived some characteristic parameters (CHPs) in the TFD, such as the quality factor $(Q)$ and center frequency $\left(f_{\mathrm{z}}\right)$. According to the experiment results, we believe that there are threshold ranges of $Q$ and $f_{\mathrm{z}}$ values in the dynamic behavior of the cardiac system for healthy projects. If the CHPs are in the proper ranges, the cardiac system is in a normal condition and homeostasis can be self-correcting. In contrast, if the values of the CHPs are less than the proper ranges, the homeostasis of the cardiac system is lost and some cardiac diseases may result. The assumption of the threshold ranges is verified by sample data from different cardiac diseases, and the results support the assumption. The CHPs are not only a kind of new reference criterion for ECG in clinical diagnosis, but also afford a technological route for the application of MCGs in cardiology.
\end{abstract}

magnetocardiography, electrocardiography, non-stationary signal, evolutionary spectrum, characteristic parameter

Citation: Liu X Y, Pei L Q, Zhang S M, et al. Characteristics of magnetocardiography and electrocardiography in the time-frequency domain. Chinese Sci Bull, 2011, 56: 819-825, doi: 10.1007/s11434-010-4066-7

The heart is the dynamic organ of the blood circulation system. The transmembrane potential changes during myocardial cells' vital processes are a biological electromagnetic signal source within living matter. Electrocardiography (ECG) and magnetocardiography (MCG), which are the carriers of electromagnetic information about the human cardiac system, can be acquired by non-invasive detection devices [1-4]. ECG and MCG signals, which are both NSS [5], have similar shapes. They both consist of a P-wave (atrial depolarization), QRS complex (ventricular depolarization) and T-wave (ventricular repolarization): the S-T segment reflects the repolarization status of myocardial cells. Transient information contained in the QRS complex

\footnotetext{
*Corresponding author (email: xinyuanliu@pku.edu.cn)
}

is extremely important for identifying changes of electromagnetic status in the cardiac system, and it can also provide clues about some cardiovascular diseases. Theoretically, in the TFD the frequency spectrum of a NSS is time-dependent, which means that traditional Fourier transform and the common wavelet transform methods are not suitable for analyzing LTFCH of those signals [6]. The evolutionary spectrum and the corresponding methods are used for detecting time-frequency domain features of NSS $[7,8]$. We have analyzed the features of the evolutionary spectrum from the QRS complex of healthy subjects' MCG and ECG signals, and then obtained the time-frequency domain characteristic parameters, that is the quality factor $Q$ and the center frequency $f_{\mathrm{z}}$. The results show that the MCG and ECG are both information carriers of cardiac electro- 
physiological activities for the same intrinsic biophysical property. However, MCG has specific advantages compared with ECG since ECG depends on the potential distribution on the body surface, which relates to the transmembrane current intensity of myocardial cells, and the latter mainly depends on the anisotropy and inhomogeneity of the myocardial tissue conductivity and the body current intensity near body surfaces. On the other hand, MCG depends on the magnetic field distribution, which is a result of the whole current, and especially on the helical current source of myocardial tissue and the intensive body current component, which results in locally intensive magnetic flux. The measurement results of over-chest magnetic fields have proved that MCG includes more important details of electromagnetic source features of the cardiac system, and especially information about the helical current source. The body surface potential does not include such details and cannot be detected by ECG. Moreover, MCG is more sensitive to the time-dependence and spatial distribution differences of the electromagnetic activities of the cardiac system [9-10]. Researchers hope that MCG, as a basic clinical diagnosis method, can predict heart diseases such as myocardial ischemia earlier than ECG. That is why so much research on the clinical application of MCGs has been done in recent years [11-13].

This paper analyzes some data from subjects with heart diseases, which originated from the MIT-BIH (Boston's Beth Israel Hospital) database. The results demonstrate that the QRS complex's CHPs for the time-frequency domain of ECG sample data vary significantly between healthy and unhealthy subjects, and that the CHPs of subjects with different heart diseases also vary statistically. According to the analyzed results of the experimental data, we propose the concept of thresholds of the QRS complex's time-frequency domain characteristic parameters for healthy subjects' ECG and MCG signals. When the CHPs are within the threshold range, the cardiac system is probably in a healthy condition, with the capability to retain homeostasis. When the CHPs are below the thresholds, the cardiac system will probably lack the capacity to be self-correcting and retain in homeostasis, and serious myocardial ischemia and other early heart diseases may follow. The threshold concept provides a new reference criterion for ECG clinical diagnosis, and a new technological route for the application of MCGs in cardiology.

\section{Evolutionary spectrum and time-frequency domain characteristic parameters of MCG and ECG}

The evolutionary spectrum is a time-dependent spectrum analysis method in the TFD based on the wavelet transform theory [14]. Firstly, the original signal is frequency modulated. A Gaussian function is used as a filter because it exhibits both well local field definition in the time domain and smoothness in the frequency domain. For the TFD, the uncertainty principle states that, for a given filter, the product of the width $\sigma_{\mathrm{f}}$ in the frequency domain and the width $\sigma_{\mathrm{t}}$ in the time domain is greater than or equal to $1 / 4 \pi$. That is $\sigma_{\mathrm{t}} \sigma_{\mathrm{f}}$ $\geqslant 1 / 4 \pi$ and $\sigma_{\mathrm{t}} \sigma_{f}=1 / 4 \pi$ for a Gaussian-shaped filter [15]. When this principle is satisfied, the three-dimensional (3D) evolutionary spectrum of a NSS, that is the local time-frequency power density distribution of a NSS in the neighborhood for an instant in time, will be obtained. Detailed ESA methods and corresponding algorithms can be found in $[7,8]$. The traditional Fourier transform describes the global frequency characteristics of a signal in the time domain. It transfers the time parameter of a signal to the frequency parameter in the frequency domain. A Fourier spectrum applies to a time-independent power spectrum and is not suitable for analyzing NSS. However, a conventional wavelet transform can be used to analyze NSS. The translational parameter and transform scale correspond to the time parameter and the frequency parameter in the TFD, respectively. As the transform scale is not the real frequency, the time and frequency resolutions of a wavelet transform vary at different points in time-frequency space [14]. The evolutionary spectrum is a time-dependent spectrum with two parameters of time and frequency, and which has the same physical interpretation as the conventional spectrum of a stationary process, in that it can describe the distribution characteristics of the power spectrum density of the signals over frequency [7,8]. The essential and crucial features of the NSS are the LTFCH in the TFD. When the uncertainty principle of the TFD is satisfied in the ESA, the time and frequency resolutions of different frequency bands are consistent; therefore, the ESA is suitable to demonstrate time-frequency domain features of NSS. Nowadays in clinical diagnosis technology, ECG data, acquired from clinical and research ECG recorders (including portable Holters), is usually classified by the QRS complex. Accordingly, this paper uses the evolutionary spectrum characteristic parameters of the QRS complex of MCG and ECG data as basic indicators to detect electromagnetic information for the cardiac system.

A QRS complex segment waveform of MCG sampled data from a healthy subject and the corresponding evolutionary spectra are shown in Figure 1. Figure 1(b) is the 3D evolutionary spectrum (3DES) computed from the QRS complex sample data with only one peak [8] (Figure 1(a)). The internal relationships among the time, frequency, and power density distributions of the QRS complex in a cardiac cycle are revealed by Figure 1(b), which contains the instantaneous electromagnetic information about the cardiac system in the neighborhood of a time instant corresponding to the R-wave. The projected image of the 3DES in Figure 1(b) onto the time-frequency $(t-f)$ plane can be seen in Figure 1(c). We select the frequency/power density spectrum distributions of half-power points range in Figure 1(b) and project to the $t-f$ plane to get Figure $1(\mathrm{~d})$, which is termed 
the half-power frequency distribution (HPFD). To further demonstrate the basic features of QRS complex evolutionary spectrum, we find the frequency $f_{\mathrm{z}}$ of the peak in Figure 1(b) and call it the center (peak) frequency. The highest frequency $\left(f_{\mathrm{H}}\right)$ and the lowest frequency $\left(f_{\mathrm{L}}\right)$ corresponding to the half-power points in Figure 1(d) are obtained. Half-power bandwidth is defined as $W_{\mathrm{b}}=\left(f_{\mathrm{H}}-f_{\mathrm{L}}\right)$. The quality factor of the evolutionary spectrum is defined as follows,

$$
Q=f_{\mathrm{z}} / W_{\mathrm{b}},
$$

where $Q, f_{\mathrm{z}}$ are the time-frequency domain characteristic parameters of the MCG evolutionary spectrum, which are the biophysical parameters of the QRS complex from the MCG (and also from the ECG).

\section{Analysis of time-frequency domain charac- teristic parameters of the MCG and ECG from healthy subjects}

The MCG and ECG data from healthy people used in this paper was obtained from the State Key Laboratory for Artificial Microstructure and Mesoscopic Physics, Physics School of Peking University. A radio frequency (RF) cardiac magnetic measurement system developed by Peking University with proprietary intellectual property rights for China is used. The RF superconducting quantum interference device (HTc rf SQUIDs) is used to acquire data from healthy subjects within the magnetic shielding room. MCG signals are collected from an over-chest 36-positions array.
Constrained by the SQUID system, an ECG signal is recorded simultaneously as MCG from a fixed lead II. The over-chest 36-positions array divides the over-chest surface into a-f(Row) $\times 1-6$ (Column) grids, and the positions are labeled as a-1, a-2, .. , a-6; b-1, b-2, .. , b-6; .. ; f-1, $\mathrm{f}-2, \ldots, \mathrm{f}-6$. This division approach is recommended by the International Biomagnetics Symposium as an international standard for MCG measurement. The peak intensity of an MCG signal is usually around 50-100 pT, while in ordinary laboratory or downtown areas, the low-frequency magnetic noise is about several $\mathrm{nT}\left(10^{-9} \mathrm{~T}\right)$, which is about 100 times larger than the human cardiac signal. Moreover, $50 \mathrm{~Hz} \mathrm{AC}$ and its harmonics can bring larger noises of up to $10^{-8} \mathrm{~T}$ or even $10^{-7} \mathrm{~T}$, which is $10^{3}-10^{4}$ times larger than the human cardiac signal. Therefore, original MCG and ECG data should be pre-processed with noise-reduction, baseline elimination and $50 \mathrm{~Hz}$ elimination before doing the evolutionary spectrum analysis. Detailed pre-process procedures are not included in this paper.

\subsection{Analysis of time-frequency domain characteristic parameters for the MCG and ECG QRS complex from the same position of different healthy subjects}

We calculated characteristic parameters $Q$ and $f_{\mathrm{z}}$ of QRS complex evolutionary spectra of MCGs from the same position e-3 and simultaneously recorded ECGs from lead II from different healthy subjects. The mean values and variance of $Q$ and $f_{\mathrm{z}}$ were obtained from the sample data from 19 healthy subjects, each of which samples lasting 36 seconds, and thus reducing time-dependence and contingency
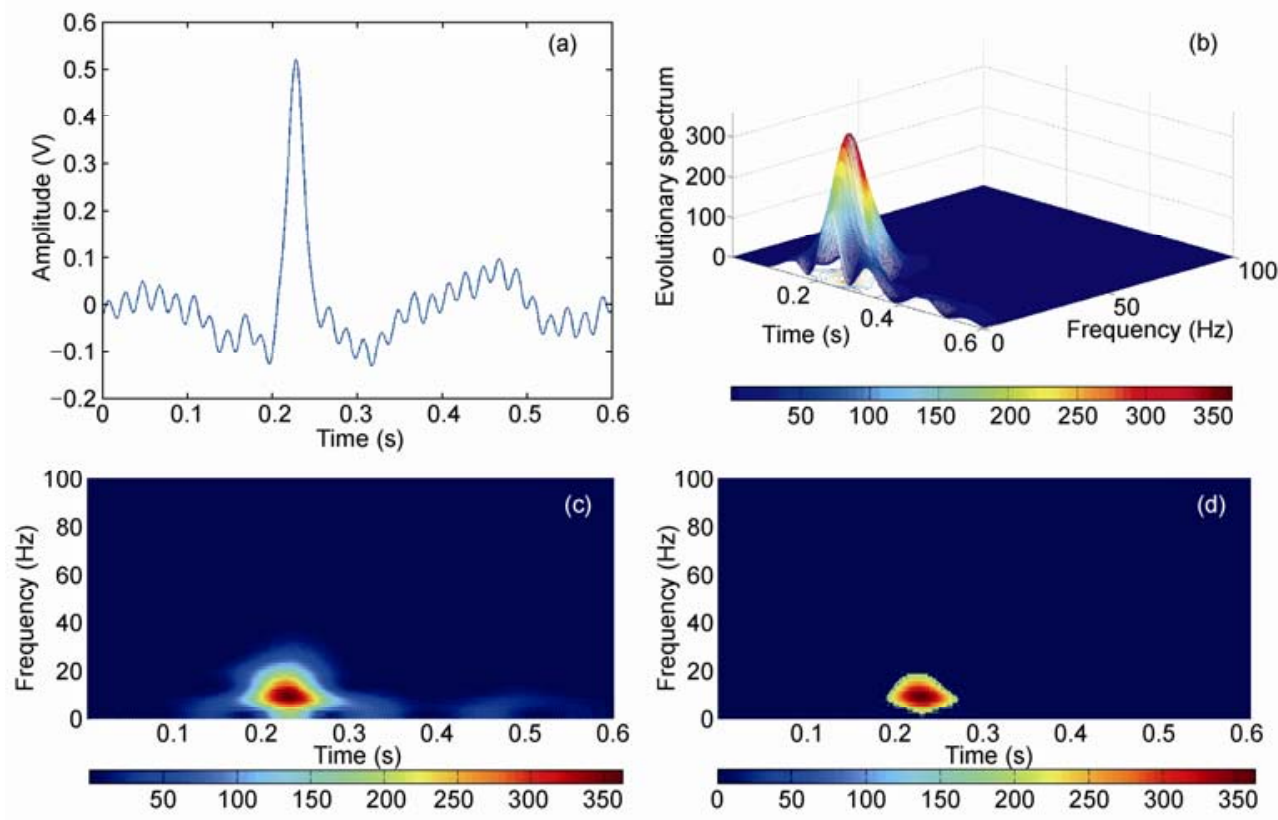

Figure 1 QRS complex segment waveform of MCG data sampled randomly from a healthy subject and the evolutionary spectra. (a) The QRS complex. (b) The 3DES of QRS complex. The coordinates time, frequency and power are denoted as $t$-axis, $f$-axis and $p$-axis, respectively. (c) The projected image of 3 DES in Figure 1(b) on the $t-f$ plane. (d) The HPFD of the QRS complex data. 
factors from the data. The results are presented in Table 1, where $Q_{\mathrm{m}}$ and $f_{\mathrm{z}-\mathrm{m}}$ are the parameters of MCG, $Q_{\mathrm{e}}$ and $f_{\mathrm{z} \text {-e }}$ are that of ECG and $\Delta Q=\left|Q_{\mathrm{m}}-Q_{\mathrm{e}}\right|$ and $\Delta f_{\mathrm{z}}=\left|f_{\mathrm{z}-\mathrm{m}}-f_{\mathrm{z} \text {-e }}\right|$ are defined as the difference values between the parameters of $\operatorname{MCG}\left(Q_{\mathrm{m}}, f_{\mathrm{z}-\mathrm{m}}\right)$ and $\operatorname{ECG}\left(Q_{\mathrm{e}}, f_{\mathrm{z}-\mathrm{e}}\right)$.

By analyzing characteristic parameters of the QRS complex evolutionary spectrum from the MCG and ECG for individual healthy subjects in Table 1 , we find the center frequency differences $\Delta f_{\mathrm{z}}=0.10-4.70$, among which $89.47 \%$ had $\Delta f_{\mathrm{z}}<3.00$. The quality factor differences $\Delta Q=0.0067-$ 0.3041 , among which $84.21 \%$ had $\Delta Q<0.1600$. Variance between different subjects is not large. The results demonstrate that the mean values of CHPs $Q$ and $f_{\mathrm{z}}$ of MCG and ECG from healthy subjects are laid in some ranges within the same time interval. For example, the quality factors $Q_{\mathrm{m}}$ and $Q_{\mathrm{e}}$ are in the range $0.5784-0.9101$, and $78.95 \%$ of $Q_{\mathrm{e}}$ and $89.47 \%$ of $Q_{\mathrm{m}}$ are distributed in the range $0.60-0.90$. The center frequency $f_{\mathrm{z}-\mathrm{m}}$ is distributed in the range 8.50 $16.00 / \mathrm{Hz}$ and $f_{\mathrm{z} \text {-e }}$ is within the range $8.00-13.90 / \mathrm{Hz}$, while $94.74 \%$ of $f_{\mathrm{z} \text {-e }}$ and $f_{\mathrm{z}-\mathrm{m}} \geqslant 9.00 \mathrm{~Hz}$. The results demonstrate that, $Q$ and $f_{\mathrm{z}}$ are representatives of the time-frequency domain characteristic parameters of the MCG and ECG, and they are characteristic indicators of the dynamic states of the human cardiac system. As MCG and ECG are data carriers of the same whole electromagnetic field of the same cardiac system, their characteristic pavameters are is determined by the intrinsic properties of the biophysical characteristics of the cardiac tissue electromagnetic fields.

\subsection{Time and spatial differences in time-frequency domain characteristic parameters of MCG and ECG QRS complex from healthy subjects}

The result of Table 1 demonstrates that the time-frequency domain characteristic parameters of MCG and ECG QRS complex from individual healthy subjects are laid in some ranges, but we can still find differences from the aspect of their distributions in time and space intervals. For example, for the time distribution, we have arranged Volunt-1's $Q$ values of position e- 3 of 36 seconds on the time axis in Figure 2(a),

Table 1 Mean values and variance of $Q$ and $f_{\mathrm{z}}$ of QRS complex of MCG and ECG data from healthy subjects ${ }^{\mathrm{a})}$

\begin{tabular}{|c|c|c|c|c|c|c|c|c|}
\hline \multirow{2}{*}{ Normal subjects } & \multicolumn{3}{|c|}{ MCG/e-3 } & \multicolumn{3}{|c|}{ ECG } & \multicolumn{2}{|c|}{ Parameter difference } \\
\hline & $Q_{\mathrm{m}}$ & $Q$-var & $f_{\mathrm{z}-\mathrm{m}}(\mathrm{Hz})$ & $Q_{\mathrm{e}}$ & $Q$-var & $f_{\mathrm{z}-\mathrm{e}}(\mathrm{H} z)$ & $\Delta Q$ & $\Delta f_{\mathrm{z}}$ \\
\hline Volunt-1 & 0.5851 & 0.0035 & 8.50 & 0.5784 & $6.22 \mathrm{e}^{-004}$ & 8.00 & 0.0067 & 0.50 \\
\hline Volunt-2 & 0.7063 & 0.0029 & 9.50 & 0.6405 & $2.27 \mathrm{e}^{-004}$ & 9.10 & 0.0658 & 0.40 \\
\hline Volunt-3 & 0.7850 & 0.0052 & 11.50 & 0.6749 & $8.48 \mathrm{e}^{-004}$ & 11.90 & 0.1101 & 0.40 \\
\hline Volunt-4 & 0.5895 & 0.0022 & 9.10 & 0.6117 & $5.99 \mathrm{e}^{-004}$ & 9.00 & 0.0222 & 0.10 \\
\hline Volunt-5 & 0.7615 & 0.0066 & 13.30 & 0.6242 & $3.08 \mathrm{e}^{-004}$ & 12.20 & 0.1373 & 1.10 \\
\hline Volunt-6 & 0.7790 & 0.0120 & 12.10 & 0.6472 & $7.31 \mathrm{e}^{-004}$ & 10.20 & 0.1318 & 1.90 \\
\hline Volunt-7 & 0.7942 & 0.0031 & 13.20 & 0.6423 & $2.66 \mathrm{e}^{-004}$ & 10.90 & 0.1519 & 2.30 \\
\hline Volunt-8 & 0.8595 & 0.0089 & 12.60 & 0.7813 & $1.30 \mathrm{e}^{-003}$ & 11.00 & 0.0782 & 1.60 \\
\hline Volunt-9 & 0.7637 & 0.0274 & 11.80 & 0.7739 & $3.10 \mathrm{e}^{-003}$ & 13.10 & 0.0102 & 1.30 \\
\hline Volunt-10 & 0.6798 & 0.0079 & 9.20 & 0.7462 & $3.96 \mathrm{e}^{-004}$ & 13.90 & 0.0664 & 4.70 \\
\hline Volunt-11 & 0.6923 & 0.0724 & 10.80 & 0.7098 & $5.93 \mathrm{e}^{-004}$ & 10.00 & 0.0175 & 0.80 \\
\hline Volunt-12 & 0.8101 & 0.0556 & 16.00 & 0.9101 & $1.10 \mathrm{e}^{-003}$ & 13.50 & 0.1000 & 2.50 \\
\hline Volunt-13 & 0.7252 & 0.0038 & 11.50 & 0.7069 & $6.29 \mathrm{e}^{-004}$ & 10.00 & 0.0183 & 1.50 \\
\hline Volunt14 & 0.7441 & 0.0320 & 15.80 & 0.6948 & $1.70 \mathrm{e}^{-003}$ & 12.70 & 0.0493 & 3.00 \\
\hline Volunt-15 & 0.8830 & 0.0163 & 13.80 & 0.5789 & $5.24 \mathrm{e}^{-004}$ & 11.00 & 0.3041 & 2.80 \\
\hline Volunt-17 & 0.7644 & 0.0063 & 13.30 & 0.5885 & $2.39 \mathrm{e}^{-004}$ & 11.00 & 0.1759 & 2.30 \\
\hline Volunt-18 & 0.8077 & 0.0062 & 12.60 & 0.6027 & $2.59 \mathrm{e}^{-004}$ & 11.00 & 0.2050 & 1.60 \\
\hline Volunt-19 & 0.6869 & 0.0271 & 13.60 & 0.6043 & $1.42 \mathrm{e}^{-004}$ & 12.00 & 0.0826 & 1.60 \\
\hline
\end{tabular}

a) In Table 1 Volunt $=$ volunteer, $Q_{\mathrm{m}}, f_{\mathrm{z}-\mathrm{m}}$ and $Q_{\mathrm{e}}, f_{\mathrm{z} \text {-e }}$ are mean values, $Q$-Var is variance, $\Delta Q=\left|Q_{\mathrm{m}}-Q_{\mathrm{e}}\right|, \Delta f_{\mathrm{z}}=\left|f_{\mathrm{z}-\mathrm{e}}-f_{\mathrm{z}-\mathrm{m}}\right|$ are parameter differences between MCG and ECG.
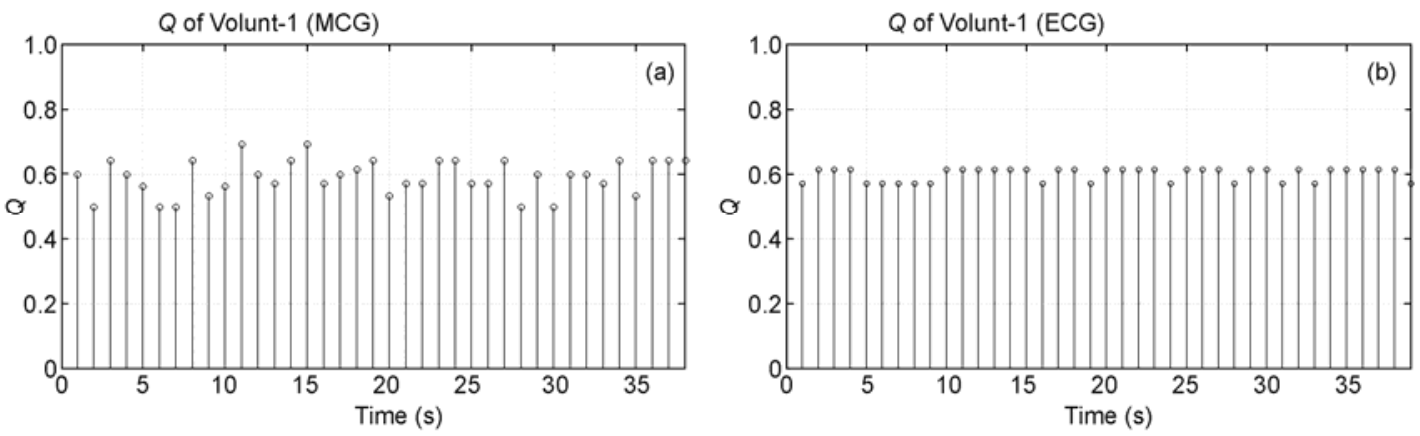

Figure 2 (a) Time distribution of $Q$ values from Volunt-1/e-3's MCG data; (b) time distribution of $Q$ values from ECG/II data. 
and the corresponding $Q$ values of ECG in Figure 2(b). The variance of MCG's $Q$ values is 0.0035 while the variance of ECG's is $6.22 \times 10^{-4}$, an order of magnitude smaller. This means that the $Q$ values of different MCG QRS complexes are more time-variable than ECG, and the MCG is more sensitive to change in the cardiac system electromagnetic field states than ECG and indicates that the electromagnetic field of the living matter in human myocardial tissue is time-dependent [5,6].

Regarding spatial distribution, our example arranges Volunt-7's $Q$ and $f_{\mathrm{z}}$ values at different positions of MCG data in the 36-positions array in Table 2. It is obvious that the values of $Q$ and $f_{\mathrm{z}}$ at different positions have certain differences, which reveals the spatial anisotropy and inhomogeneity of conductivity in living matter of the cardiac tissue. The spatial mean value of $Q$ is 0.7614 , and the mean value of $f_{\mathrm{z}}$ is $13.37 \mathrm{~Hz}$ in Table 2, while Volunt-7/e-3's time mean value of $Q$ is 0.7942 , and the mean value of $f_{z}=13.20$ $\mathrm{Hz}$ in Table 1: these are consistent. That is also why we chose data of MCG/e-3 in Table 1.

According to the results above, we have a proposal as following: the characteristic parameters of the MCG and ECG QRS complexes of healthy subjects are laid in some ranges, and have recommended the ranges of values which are for $Q=0.60-0.90$ and for $f_{\mathrm{z}} \geqslant 9.00 \mathrm{~Hz}$. These thresholds are some of the basic criteria in detecting normal or abnormal status of the cardiac system electromagnetic field. When $Q$ and $f_{\mathrm{z}}$ are out of the proper ranges, the cardiac system probably exhibits a lack of capacity of self-correcting or a lack of remaining in homeostasis. This may cause some serious myocardial ischemia or other early heart diseases. We have verified the rationality of the proposed ranges using the data extracted from the MIT-BIH database.

\section{Verifying the thresholds concept of charac- teristic parameters using ECG sample data from unhealthy subjects}

3.1 Analysis of characteristic parameters in the time-frequency domain of ECG QRS complex data from unhealthy subjects with different heart diseases

To verify the rationality of our concept of the proposed thresholds, we chose the ECG data of unhealthy subjects from the MIT-BIH database. Firstly, we chose data from the Long-Term ST Database in the MIT-BIH database (http:// www.physionet.org) to do statistical analysis on diverse samples. This data includes 80 ECG sample data of coronary heart disease, angina pectoris and early myocardial infarction: each of these samples lasts 21-24 hours and includes signals from 2 or 3 leads, with a sample rate of 250 $\mathrm{Hz}$ and a sample resolution of 12 bits. Each sample of data was independently identified by three experts, and then added to the database only if an agreement was reached, and so guaranteeing the reliability of the data. The results of the characteristic parameters of ECG QRS complex in the time-frequency domain from different heart diseases are shown in Figure 3.

Of all the coronary heart diseases included in Figure 3, 13 subjects are recorded from V4 and MLIII, among which $15 \%-23 \%$ have a $Q<0.60$, and about $77 \%$ have an $f_{\mathrm{z}}<9.00$ Hz. 15 subjects are recorded from V6, aVF and V1, among which from V6 and aVF about $33 \%$ have a $Q<0.60$, and from V1 about $86 \%$ have a $Q<0.60$, while from V1, V6 and aVF $80-93 \%$ have an $f_{\mathrm{z}}<9.00 \mathrm{~Hz}$. Of the angina pectoris subjects, 12 subjects are recorded from V6, aVF and V1, among which from V6 and aVF $25 \%-30 \%$ have a $Q<0.60$, and from V1 about $75 \%$ have a $Q<0.60$, while $72-100 \%$ have an $f_{\mathrm{z}}<9.00 \mathrm{~Hz} .20$ subjects are recorded from V4 and MLIII, among which about $15 \%$ have a $Q<0.60$, while $70 \%-80 \%$ have an $f_{\mathrm{z}}<9.00 \mathrm{~Hz}$. Of those with early myocardial infarction, five subjects are recorded from V4 and MLIII, among which about $20 \%$ have a $Q<0.60$, while about $80 \%$ have an $f_{z}<9.00 \mathrm{~Hz}$. The results demonstrate that, of all the time-frequency domain characteristic parameters of the ECG QRS complex from all leads of different heart disease subjects, whenever the $Q$ value is below the threshold, then the corresponding $f_{\mathrm{z}}$ will also be below its threshold. Moreover, $Q$ and $f_{\mathrm{z}}$ are not sensitive to age or gender. Of all the different heart diseases, the percentage of subjects' $f_{\mathrm{z}}$ which are significantly below threshold $9.00 \mathrm{~Hz}$ is 70\%-93\%. Although the percentage of subjects with $Q<0.60$ varies significantly when the leads and diseases are different, the trend of $Q \mathrm{~s}$ and $f_{\mathrm{z}} \mathrm{s}$ in different diseases demonstrates that the threshold concept of characteristic parameters in the ECG QRS complex evolutionary spectrum of healthy subjects (of $Q=0.60-0.90$ and $f_{\mathrm{z}} \geqslant 9.00 \mathrm{~Hz}$ ) is basically correct. It should be noted that coronary heart disease, angina pectoris and early myocardial infarction are all

Table 2 Volunteer 7's $Q$ and $f_{\mathrm{z}}$ distribution of MCG QRS evolutionary spectrum at different positions

\begin{tabular}{|c|c|c|c|c|c|c|c|c|c|c|c|c|}
\hline \multirow[b]{2}{*}{ Positions } & \multicolumn{6}{|c|}{ MCG's $Q$ values of different positions } & \multicolumn{6}{|c|}{ MCG's $f_{z}$ values of different positions } \\
\hline & 1 & 2 & 3 & 4 & 5 & 6 & 1 & 2 & 3 & 4 & 5 & 6 \\
\hline $\mathrm{a}$ & N/A & N/A & N/A & N/A & N/A & N/A & N/A & N/A & N/A & N/A & N/A & N/A \\
\hline $\mathrm{b}$ & N/A & N/A & N/A & 0.8994 & 0.8606 & N/A & N/A & N/A & N/A & 16.47 & 16.05 & N/A \\
\hline $\mathrm{c}$ & N/A & 0.6667 & 0.6217 & 0.6393 & 0.6393 & N/A & N/A & 9.22 & 8.52 & 14.61 & 14.61 & N/A \\
\hline d & N/A & 0.6757 & 0.6824 & 0.5973 & N/A & N/A & N/A & 11.06 & 10.68 & 9.45 & N/A & N/A \\
\hline e & N/A & 0.7796 & 0.7644 & 0.8385 & 0.7804 & N/A & N/A & 12.63 & 13.32 & 14.73 & 15.95 & N/A \\
\hline
\end{tabular}



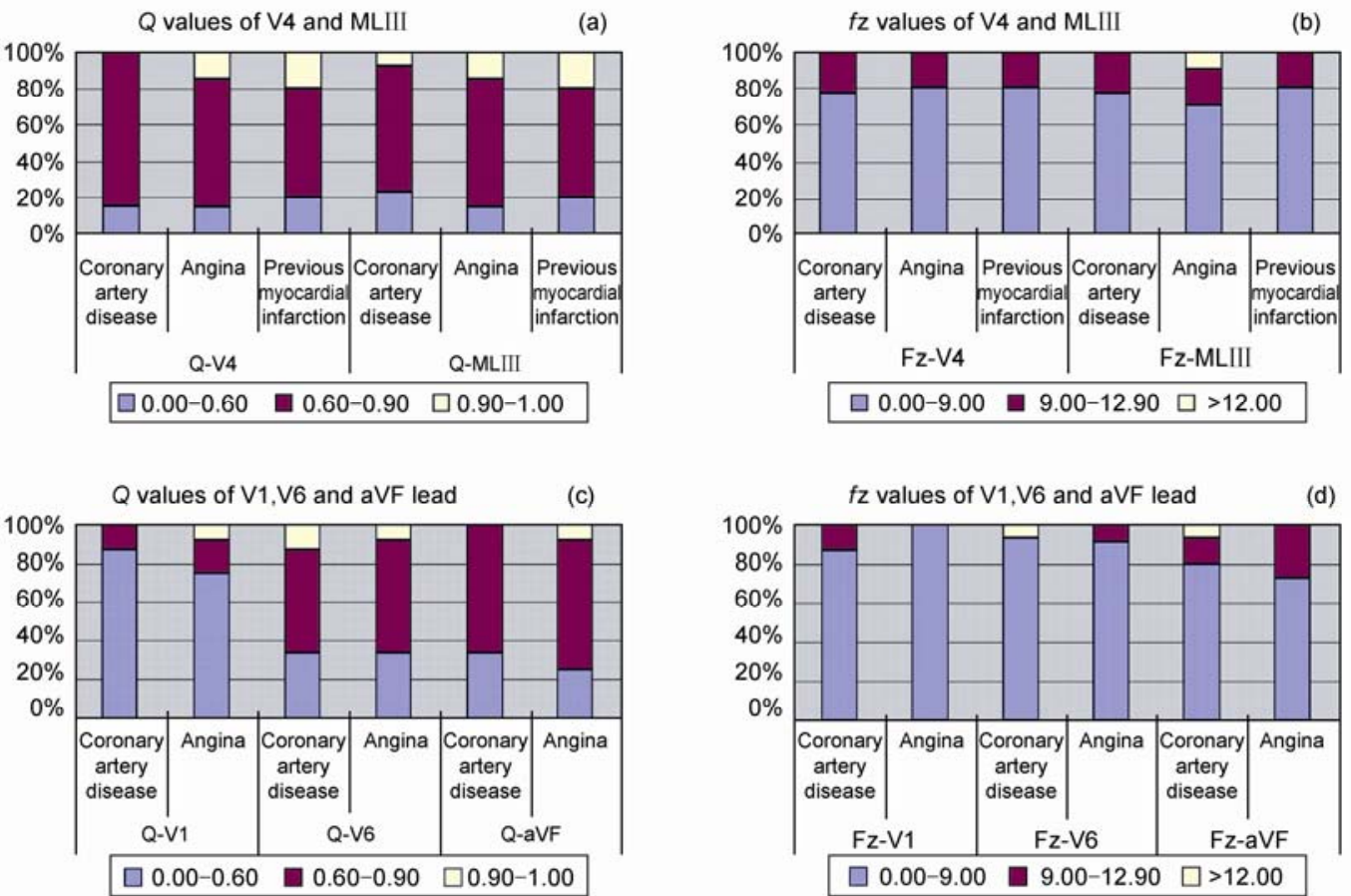

Figure 3 Results of time-frequency domain characteristic parameters of ECG QRS complex from different heart diseases. (a) $Q$ values of coronary heart disease, angina pectoris and early myocardial infarction, V4, ML III lead. (b) $f_{z}$ values of coronary heart disease, angina pectoris and early myocardial infarction, V4, ML III lead. (c) $Q$ values of coronary heart disease, angina pectoris and early myocardial infarction, V1,V6, aVF lead. (d) $f_{z}$ values of coronary heart disease, angina pectoris and early myocardial infarction, V1,V6, aVF lead. Units of $f_{z}$ in (b) and (d) is Hz. Threshold range of a healthy subject's $Q$ value is $0.60-0.90$, and center frequency $f_{\mathrm{z}} \geqslant 9.00 \mathrm{~Hz}$

early heart diseases which may result in fatal diseases such as myocardial infarction or sudden cardiac death. However, patients with early myocardial ischemia usually have no obvious symptoms, and the ECG might also seem normal: even some patients before myocardial infarction would produce seemingly normal ECGs after angina pectoris, thus resulting in delayed diagnosis and treatment. In this paper the percentage of subjects' $f_{\mathrm{z}}$ significantly below the threshold of $9.00 \mathrm{~Hz}$ is $70 \%-93 \%$ of all different heart diseases, and this may therefore become a forewarning indicator of early heart diseases.

\subsection{Analysis of time-frequency domain characteristic parameters of ECG data in sudden cardiac death}

In order to analyze the changing process and features of the characteristic parameters through time in sudden cardiac death, we chose part of No.52 ECG data from the Sudden Cardiac Death Holter Database, MIT-BIH database. The data was from two leads which were marked as Chan-1 and Chan-2, lasted 7 hours 15 minutes and time intervals 1 minute long and 15 minutes apart were used. Figure 4 demonstrates the mean values of $Q$ and $f_{\mathrm{z}}$ at different time intervals recorded by Chan-1 and Chan- 2 .

Figure 4 demonstrates that, first of all, $Q$ values of Chan- 1 and Chan- 2 follow the same trend throughout the time axis. In Chan- $1,80.65 \%$ of $Q$ is less than 0.60 , and in
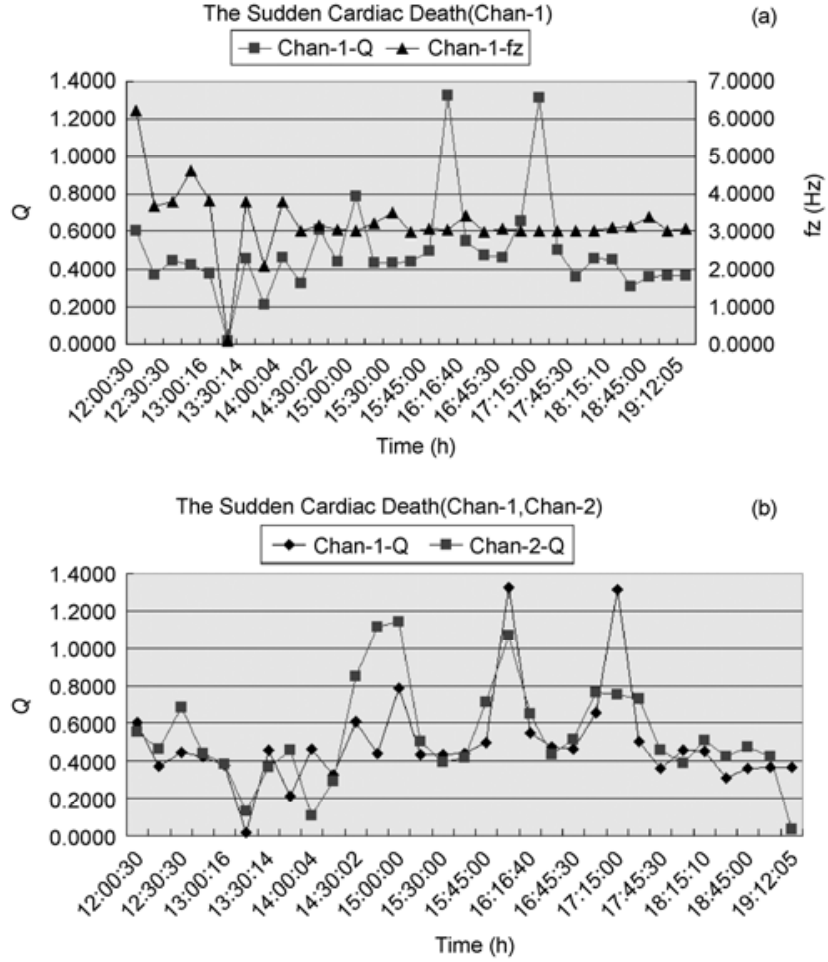

Figure 4 (a) $Q$ values of two leads ECG data of NO.52 patient; (b) $Q$ and $f_{\mathrm{z}}$ of Chan-1 lead of No.52 patient.

Chan-2, $67.74 \%$ of $Q$ is less than 0.60 . All the data has 
pathological features (compare it with Figure 4(b)). Secondly, the center frequency $f_{\mathrm{z}}$ of Chan- 1 and Chan- 2 are both below threshold $(9.00 \mathrm{~Hz})$, and whenever the $Q$ value is below the threshold, $f_{\mathrm{z}}$ is also below the threshold. In Figure 4(b), both $Q$ and $f_{\mathrm{z}}$ tend to decrease over time. As time passes, the center frequency $f_{\mathrm{z}}$ tends to fall consistently, which means it is capable of reflecting earlier pathological changes in the cardiac system. In Figure 4(a), the $Q$ values fluctuate, and the fluctuation trends of the two leads' $Q$ values are almost consistent, indicating some changes of the homeostasis capacity in the cardiac system. This example demonstrates that the threshold concept of characteristic parameters of ECG data has rationality and the threshold concept can also apply to MCG data.

\section{Conclusions}

This paper analyzed the evolutionary spectrum of QRS complex of MCG and ECG data from healthy subjects, and the results demonstrated that MCG and ECG characteristic parameters of individual healthy subjects are laid in some ranges. However, MCG has differences from ECG. MCG is more sensitive to the time-dependence and spatial distribution differences in the electromagnetic activities of a cardiac system, so MCG may include more important details of the features from the electromagnetic field sources in a cardiac system. MCG and ECG are like Ballet's "two toes"; that is, although they have differences in time and space, as information data carriers of the same cardiac system electromagnetic field, they have similarly the time-frequency domain features, which are determined by the intrinsic biophysical property for the instant electromagnetic behaviors of the cardiac system of the "Dancer".

The present study proposes that, in the cardiac dynamics, the time-frequency domain characteristic parameters $Q$ and $f_{\mathrm{z}}$ are laid in some ranges and both have thresholds. When the characteristic parameters are within the threshold, the cardiac system is probably in a healthy condition with the capacity of homeostasis. When the characteristic parameters are below the threshold, the cardiac system probably lack the capacity of self-correcting and retaining in homeostasis, and serious myocardial ischemia or other early heart diseases are likely to ensue. This paper tested the evolutionary spectrum characteristic parameters of ECG signals from healthy and unhealthy subjects, and it established that the threshold ranges in the characteristic parameters exist. We recommend threshold values of healthy subjects to be: quality factor $Q=0.60-0.90$ and center frequency $f_{\mathrm{z}} \geqslant 9.00 \mathrm{~Hz}$. The threshold concept affords the basic criteria for detecting normal or abnormal status of the cardiac system electromagnetic field. This is an experimental/empirical conclusion from experiment results, so $Q$ and $f_{\mathrm{z}}$ are the biophysical parameters of QRS complex, while the physiological meaning is not so far clear. The reliability of the threshold concept should be further verified by using the evolutionary spectrum method to analyze more experimental and clinical data, especially to large amounts of clinical MCG and ECG data, and then further modifying our assumption. Only when doctors' clinical experience is combined with the threshold concept may technical parameters helpful to clinical medicine be achieved.

This work was supported by the National Natural Science Foundation of China (607710003). The authors would like to thank Professor Ping Ma, Physics School of Peking University for original MCG and ECG data support of healthy subjects, and thank MIT-BIH database for partial ECG data of heart disease patients.

1 Holter N J. New methods for heart studies. Science, 1961, 134: 1214

2 Baule G M, McFee R. Detection of the magnetic field of the heart. Am Heart J, 1963, 66: 95

3 Cohen D, Edelsack E A, Zimmerman J E. Magnetocardiograms taken inside a shielded room with superconduction point-contact magnetometer. Appl Phys Lett, 1970, 16: 278

4 Karsten S, Alex I. Braginski, biomagnetism using SQUIDs: Status and perspectives. Supercond Sci Technol, 2006, 19: S160-S170

5 Pola A, Macerata A, Emdin M. Estimation of the power spectral in nonstationary cardiovascular time series: Assessing the role of the time-frequency representations (TFR). IEEE Trans Biomed Eng, 1996, 43: 46-59

6 Friedman B H, Allen M T, Christie I C, et al. Validity concerns of common heart-rate variability indices addressing quantification issues in time- and frequency-domain measures of HRV. IEEE Eng Med Bio, 2002, 21: 35-40

7 Priestley M B. Wavelets and time-dependent spectral analysis. J Time Series Analysis, 1996, 17: 85-103

8 Liu X, He D, Pei L, et al. Singularity detection of the rabbit electrocardiogram: An evolutionary spectral method. Prog Nat Sci, 2009, 19: $1317-1326$

9 Liu X, Xie B, Dai Y, et al. Investigation on the adaptive filter of magnetocardiography data from radio frequency SQUID. Acta Phys Sin, 2005, 54: 1937-1942

10 Kosch O, Meindl P, Steinhoff U, et al. Physical aspects of cardiac magnetic fields and electric potentials. In: The 12th International Conference on Biomagnetism, Biomag 2000, August 13-17, 2000 Helsinki, Finland (http://biomag2000.hut.fi/papers_all.html)

11 Goernig M, Liehr M, Tute C, et al. Magnetocardiography based spatiotemporal correlation analysis is superior to conventional ECG analysis for identifying myocardial injury. Inter J Bioelectromagnetism, 2009, 11: $107-111$

12 Wang H W, Ding H S, Zhang L H, et al. A high-Tc dc-SQUID-based single-channel MCG and its performance. Chinese Sci Bull, 2002, 47: 1861-1864

13 Li Y J, Yan H, Wang Z L, et al. Study of electrocardio-waveform variability. Sci China Ser C: Life Sci, 2009, 39: 1181-1187

14 Daubbechies I. Ten Lectures on Wavelets. Philadelphia: Society for Industrial and Applied Mathematics, 1992

15 Gabor D. Theory of communication. Proc Inst Elect Eng, 1946, 93: $429-457$

Open Access This article is distributed under the terms of the Creative Commons Attribution License which permits any use, distribution, and reproduction in any medium, provided the original author(s) and source are credited. 